\title{
Un gène responsable de la maladie de Usher de type 1, repéré sur le chromosome 14 : des souris et des hommes...
}

La maladie de Usher désigne une association surdité et cécité d'origine génétique. C'est une affection récessive autosomique. Elle touche environ un nouveau-né sur 30000 , rend compte d'environ 5 à $6 \%$ de l'ensemble des surdités infantiles [1] et $15 \%$ des rétinites pigmentaires [2]. Il en existe au moins deux sous-groupes cliniques [3]. C'cst un des gènes responsables de la forme grave (type I) qui vient d'être localisé sur le bras long du chromosome 14. Cette forme sévère associe : (1) une surdité congénitale profonde interdisant l'acquisition du langage ; (2) un déficit vestibulaire total responsable d'un retard à l'acquisition de la marche et de troubles de l'équilibre et (3) une rétinopathie pigmentaire débutant vers l'âge de 10 ans et évoluant rapidement, faisant de ces enfants des sourds-aveugles. Il existe une forme modérée de cette maladie (type II) associant une hypo-acousic moins importante permettant le langage et une rétinopathie pigmentaire plus tardive et moins sévère. Le groupe de Kimberling avait repéré, en 1990, le gène responsable de ce type II sur le chromosome 1 [4] (m/s $n^{\circ} 7$, vol. 8, p. 740). Les travaux sur la localisation du gène responsable de la forme grave de la maladie de Usher ont commencé à l'automne 1990 avec la collaboration de la consultation d'ophtalmologie de l'hôpital Laënnec, après qu'un étudiant au DEA de Génétique de Paris, le docteur Dominique Bonneau, originaire de Poitiers cut remarqué et recensé un grand nombre de familles éprouvées par cette maladie dans les Institutions de sourds de Poitiers.

Nous avons tout d'abord testé et éliminé l'hypothèse d'une forme allélique du type II confirmant l'hétérogénéité génétique entre les deux sous-groupes cliniques [5]. Puis, en août 1991, pour le $11^{\mathrm{e}}$ Séminaire International de Cartographie Génétique de Londres, nous présentions la localisation du gène responsable du type I sur le bras long du chromosome 14 en montrant une liai- son génétique entre le gène morbide ségrégeant dans 13 familles et plusieurs marqueurs d'ADN polymorphes de la région $14 q 32$ [6].

Cette localisation ne fut pas retrouvée dans les familles américaines, prouvant, comme c'était connu pour d'autres maladics, que plus d'un gène pouvait rendre compte d'une même affection héréditaire. Après un lourd travail d'études de nouvelles familles, nous parvenions à confirmer la localisation sur le chromosome 14 dans certaines familles et à l'exclure dans d'autres [7]. Le point original de ces travaux est que la quasi-totalité des familles dans lequel cette localisation fut confirmée était originaire de la région PoitouCharentes et particulièrement du département des Deux-Sèvres. Il est vraisemblable qu'un effet fondateur à partir d'un même ancêtre explique cette origine commune et la fréquence de la maladic de Usher dans cette région.

Très récemment, l'équipe de Kimberling localisait sur le bras long du chromosome 11 [8] un gène responsable de la maladie dans d'autres populations (États-Unis, Suède, Irlande et Afrique du Sud) tandis que le groupe de Smith excluait cette localisation dans les familles acadiennes de Louisiane et trouvait un troisième locus sur le bras court du chromosome 11 [9], spćcifique de cette population d'origine française, mais non poitevine (originaire de Normandie et de Bretagne). Il est de la plus grande importance de bien noter que les deux localisations (11q et $11 p)$ furent exclues dans toutes nos familles poitevines liées au chromosome 14 , et que la localisation en $11 \mathrm{p}$ fut exclue dans l'ensemble de nos familles, liées et non liées au 14. En revanche, et ces résultats sont naturellement préliminaires, il semble bien que le gène repéré par Kimberling sur le bras long du chromosome 11 pourrait rendre compte de la maladie dans les familles françaises non poitevines (résultats suggestifs, étude en cours). Les conséquences de notre localisation sur le chromosome 14 sont très importantes.

Effectivement, on sait peu de choses de la pathogénie du syndrome de Usher. L'hypothèse la plus séduisante est celle d'une anomalie de la fonction ciliaire pour expliquer l'atteinte combinée de trois systèmes sensoriels différents. Effectivement, les photorécepteurs, les cellules de l'oreille interne et les cellules vestibulaires se développent à partir de cellules progénitrices ciliées et plusieurs publications mettent en lumière des anomalies des axonèmes chez des patients atteints de syndrome de Usher [8, 9].

Par ailleurs, le gène du situs inversus dont on connaît la corrélation avec une fonction ciliaire déficiente, a été localisé en 1986 sur le chromosome 12 chez la souris [10]. Il est particulièrement intéressant de noter que la région du chromosome où paraît être localisé un des gènes de Usher type I est la région homologue du chromosome 12 murin. Ainsi l'hypothèse d'une batterie de gènes jouant un rôle dans la fonction ciliaire paraît probable et demande à être explorée. Si elle se confirme, elle révélera un second mécanisme de survenue des rétinopathies pigmentaires, après l'anomalie de la cascade de la phototransduction.

$$
\text { J.K., D.B., A.M. }
$$

1. Boughman JA, Vernon $M$, Shaver KA. Usher syndrome : definition and estimate of prevalence from two high-risk populations. J Chronic Dis 1983 ; 36 : 595-603.

2. Kaplan J, Bonneau D, Frezal J, Munnich A, Dufier JL. Clinical and genetic heterogeneity in retinitis pigmentosa. Hum Genel 1990; 85 : 635-42.

3. Fishman GA, Kumar A, Joseph ME, Torok N, Anderson RJ. Usher's syndrome : ophtalmic and neuro-otologic findings suggesting genetic heterogeneity. Arch Ophtalmol 1983; 101 : 1367-74.

4. Kimberling WJ, Weston MD, Moller C, et al. Localization and Usher syndrome type II to chromosome 1q. Genomics 1990; 7 : 245-9.

5. Kaplan J, Guasconi G, Bonneau D, et al. Usher syndrome type $I$ is not linked to D1S81 (pTHH33) : evidence for genetic heterogeneity. Ann Genet 1990 ; 33 : 105-8. 
6. Kaplan J, Gerber S, Bonneau D, et al. Iocation of Usher type I gene on chromosome 14q by linkage with D14S13 (MIJJ14 probe). 11th International Workshop on Human Gene Mapping, London, 18-22 August, 1991.

7. Kaplan J, Gerber S, Bonneau D. A gene for Usher syndrome type I gene maps to chromosome 14q. Genomics 1992; 4 : 979-87.

8. Kimberling WJ, Moller CG, Davenport S, et al. Linkage of Usher syndrome type 1 gen (USH1B) to the long am of chromosome 11 Genomics $1992 ; 14: 988-94$

9. Smith RJA, Lee EC, Kimberling WJ, at al. Localization of two genes for Usher syndrome type I to chromosome 11, Genomics 1992; 14 995-1002.

10. Hunter DG, Fishman GA, Mehta RS, Kretzer FL. Abnormal sperm and photoreceptor axonemes in Usher's syndrome. Arch Ophealnol 1986 104: 385-9.

11. Bonneau D, Raymond F, Kremer C, Klossek JM, Kaplan J, Patte F. Usher syndrome type I associated with bronchectiasis and immobile nasal cilia in two brothers. J Med Genet 1993 (sous presse)

12. Brueckner M, D'Eustachio P, Horwich AL. Linkage mapping of a mouse gene, "iv " tha controls left-right asymmetry of the heart and viscera. Proc Nall Acad Sci USA 1989 ; 86 : 5035-8.

Un colloque international sur le thème "Anxiété : neurobiologie, clinique et perspectives thérapeutiques se tiendra les 20 et 21 septembre 1993 à Dinard (llle-et-Vilaine) avec, entre autres, le soutien de I'INSERM

Organisateurs : M. Hamon et M.-H. Thiebot (INSERM U.288), H. Ollat (ANPP-Association pour la Neuro-Psycho-Pharmacologie)

Au programme : Aspects cliniques des différentes formes d'anxiété pathologique ; Modèles animaux ; Comportements sociaux lagression, défense) ; Implications des systèmes mono-aminergiques, GABA-ergiques, et autres ; Implications neuro-endocriniennes et immunitaires: Mécanismes d'action des anxiolytiques (aspects moléculaires et pharmacologiques des récepteurs du GABA, de la sérotonine, de la cholécystokinine, des acides aminés excitateurs, etc.) ; Nouvelles perspectives thérapeutiques.

Conférenciers : R. Balster (Richmond, USA) J.-C. Baron (INSERM U.320, Caen), G. Biggio (Cagliari, Italie), J.-Ph. Boulanger (INSERM Sherbrooke, Canada), M. Bourin (Nantes), R. Dantzer (INSERM U.176, Bordeaux), V. Daugé (INSERM U.266, Paris), A. Depaulis (INSERM Strasbourg), H. Gozlan (INSERM U.2B8, Paris), W. Haefely (Bàle, Suisse), F. Héry (INSERM U.297, Marseille), H. Lüddens (Heidelberg, Allemagne), C.A. Marsden (Nottingham, G.B.), M.-J. Millan (Puteaux), D. Sanger (Bagneux), J.-P. Tassin (INSERM U.114, Paris), M.-H. Thiebot (INSERM U.288, Paris).

Ce colloque réunira une centaine de participants. Les actes paraitront pour le colloque. sous forme d'une cototion INSERM/John Libbey Eurotext dans la collection des Colloques do I'INSERM.

Date limite d'inscription : 15 mars 1993

Pour tout renseignement complémentaire et proposition de communication affichée s'adresser à l'A.N.P.P., 25, rue de la Plaine, 75020 Paris - Tél. : 43.79.95.42 - Télécopie : 43.79.34.34

\section{BRÈVES}

Un nouveau gène de résistance aux produits cytolytiques. Cole et al. (Kingston, Ontario, Canada) viennent de décrire un nouveau membre de la super-famille des transporteurs membranaires dépendant de l'ATP. L'un des mécanismes de la résistance des cellules cancéreuses aux produits cytoliques ou cytostatiques est l'hyperexpression du gène $M D R 1$ (multi drug resistance). Ce gène est souvent amplifié dans les cellules néoplasiques soumises à une chimiothérapie anti-cancéreuse. Le produit du gène $M D R 1$, la $\mathrm{P}$ glycoprotéine, est un transporteur membranaire qui semble capable d'expulser hors de la cellule les substances anti-mitotiques par un mécanisme consommant de l'énergie. Cette protéine appartient à une super-famille de transporteurs membranaires possédant tous un domaine de liaison de l'ATP (domaine $A B C, A T P$-binding cassette). Le produit du gène de la mucoviscidose, le CFTR (cystic fibrosis transmembrane regulator) et des transporteurs de peptides se liant à des molécules de classe 2 du complexe majeur d'histocompatibilité (gènes TAP) appartiennent également à cette famille. L'hyperexpression de la P-glycoprotéine et l'amplification du gène $M D R 1$ sont des phénomènes rares dans le cancer du poumon. La lignée cellulaire H69 est dérivée d'un cancer du poumon à petites cellules et a été très intensément utilisée pour étudier les caractéristiques de ce type de prolifération. Une variante résistante aux substances chimiothérapiques a été appelée H69 AR. Une banque d'ADN complémentaire des cellules variantes H69 AR a été construite et soumise à un criblage différentiel utilisant des sondes dérivées de cel- lules $\mathrm{H} 69$ et $\mathrm{H} 69$ AR. Ainsi a pu être caractérisé un ADN complémentaire caractéristique des cellules résistantes, hybridant avec un ARN messager de 7,8 à $8,2 \mathrm{~kb}$. La séquence codante complète de ce messager indique qu'il possède un domaine $A B C$ de liaison de l'ATP, et semble donc appartenir, lui aussi, à cette super-famille des transporteurs membranaires. Cependant, le reste de sa séquence est extrêmement éloigné de celle des autres membres de cette famille. Alors que les gènes MDR1 et CFTR sont localisés sur le bras long du chromosome 7, ce nouveau gène, appelé $M R P$ (multi-drug resistance-associated protein) est situé en 16 p 13.1. Les auteurs n'ont pas prouvé que le produit du gène $M R P$ était la cause de la résistance des cellules $\mathrm{H} 69$ $\mathrm{AR}$ aux anti-mitotiques. Cependant, une bonne corrélation existe entre l'amplification du gène $M R P$ et la résistance à ces substances. Le mécanisme précis d'action de la protéine MRP n'est pas clair, lui non plus, puisque la concentration intracellulaire des antimitotiques n'est pas diminuée dans des cellules résistantes par rapport aux cellules sensibles. Les auteurs suggèrent, mais, là encore, sans preuve, que la protéine MRP pourrait intervenir dans la localisation subcellulaire de ces produits. Enfin, une hyper-expression de cette protéine a aussi été observée ex vivo, dans des cellules cancéreuses établies (cellules HeLa dérivées d'un cancer du col utérin) et in vivo, dans des cancers pulmonaires " non à petites cellules".

[1. Marie JP. mádecine/sciences 1990 ; 6 : 443-8.]

[2. Cole SPC, et al. Scince $1992 ; 258$ : 1650-4.]

\section{INTÉRÊT DES CULTURES CELLULAIRES ANIMALES ET HUMAINES EN PHARMACOTOXICOLOGIE}

\section{du 15 au 26 mars 1993}

Cycle d'études organisé par le laboratoire de Pharmacologie Cellulaire de l'EPHE. Ce stage rentre dans le cadre de la Formation Permanente et s'adresse aux chercheurs et techniciens scientifiques du secteur privé et public.

Responsable scientifique : Madame Monique Adolphe - Laboratoire de Pharmacologie Cellulaire, 15, rue de l'École de Médecine • 75006 Paris - Tél. : 43.29.28.69 • Fax 44.07.10.52 
口 Cardiomyopathie hypertrophique sporadique et mutations de novo de la myosine. Le groupe de J. G. et C. E. Seidman (Boston, MA, USA) a découvert en 1990 que la cardiomyopathie hypertrophique familiale, affection transmise selon le mode autosomique dominant, était liée, dans la moitié des cas environ, à une mutation de la chaîne lourde $\beta$ de la myosine cardiaque $\left(\mathrm{m} / \mathrm{s} n^{\circ} 9\right.$, vol. 6, p. 920). Un total de sept mutations a été trouvé, toutes du type faux-sens, et toutes situées dans la moitié amino-terminale de la molécule [1]. Cette même équipe [2] a analysé ce gène chez sept malades atteints de la forme sporadique de la maladie. Dans deux cas, des mutations furent trouvées, montrant que des mutations peuvent survenir de novo. Un des sujets mutés a transmis la maladie à un descendant, indiquant qu'il devait s'agir d'une mutation de la lignée germinale. L'apparition simultanée, dans une famille, d'une cardiomyopathie et d'une mutation d'une chaîne lourde de la myosine est un argument probant en faveur du rôle causal des altérations de la myosine dans la genèse de la maladie.

[1. Watkins $\mathrm{H}$, et al. $N$ Engl J Med 1992 ; 326 : 1108-14.]

[2. Watkins $\mathrm{H}$, et al. J Clin Invest 1992 ; 90 : 1666-71.]

Ataxie-télangiectasie, instabilité génomique et p53. La protéine p53 bloque en $\mathrm{G} 1$ le cycle cellulaire de cellules dont l'ADN a été endommagé $\left(\mathrm{m} / \mathrm{s} \quad n^{\circ} 9\right.$, vol. 8 , p. 1002). Lorsque des fibroblastes normaux sont soumis à des radiations ionisantes, même à des doses entraînant peu de lésions de l'ADN, ils cessent de proliférer alors qu'augmente l'expression des gènes p53 et GATT45. Dans l'ataxie-télangiectasie, une maladie humaine autosomique récessive caractérisée par une ataxie cérébelleuse progressive avec dégénérescence des cellules de Purkinje, une hypersensibilité aux radiations ioni- les fibroblastes en culture sont remarquables par l'insensibilité de leur synthèse d'ADN à l'irradiation. Ce phénomène est parallèle à une nonactivation des gènes $p 53$ et GATT 45 lors d'un traitement aux radiations ionisantes. Cependant, le transfert dans ces cellules d'un vecteur d'expression commandant l'accumulation de protéine p53 entraîne une activation du gène GATT45 et un blocage du cycle cellulaire en G1. Par conséquent, M.D. Kastan, et al. (Baltimore, MD ; Bethesda et Cambridge, MA, USA) proposent un schéma dans lequel le gène de l'ataxie-télangiectasie (AT) est indispensable à la transmission du signal induit par les radiations ionisantes, et donc à l'activation du gène p53. La protéine p53 peut se fixer en un site intronique du gène GATT45 dont elle est, très probablement, un activateur transcriptionnel. Le produit de ce dernier gène serait alors, directement ou indirectement, responsable du blocage des cellules en G1 [1]. L'absence d'induction de p53 et de GATT45 faciliterait la fixation et l'accumulation de lésions de l'ADN et jouerait donc un rôle dans la susceptibilité de ces malades au cancer. [1. Kastan MD, et al. Cell $1992 ; 71$ : 587-97.]

Le knock-out du gène de l'inhibine est associé au développement de tumeurs gonadiques. Les inhibines sont des hétérodimères $\alpha / \beta$ composés de sous-unités appartenant à la même famille que le $\mathrm{TGF} \beta$, l'hormone anti-müllérienne et des inducteurs de la différenciation mésodermique chez Xenopus. L'action des inhibines est antagoniste de celle des activines (dimères de structure générale $\beta / \beta$ ) sur la sécrétion de FSH par l'hypophyse et la gamétogenèse. Chez la femelle, l'inhibine entraîne une atrésie folliculaire et inhibe la prolifération des cellules de la granulosa alors que l'activine a un effet inverse. L'équipe de A. Bradley (Houston, TX, USA) vient de montrer que les mâles et les femelles homozygotes pour une mutagenèse insertionnelle du gène de l'inhibine $\alpha$ étaient stériles et développaient des tumeurs gonadiques, ovariennes et testiculaires, accompagnées d'une prolifération de tissu stromal mal différencié avec d'importants remaniements tissulaires. Dans les testicules, la spermatogenèse est bloquée parallèlement au développement de la tumeur et la proportion des cellules de Leydig est diminuée, suggérant la participation des cellules de Sertoli au processus tumoral. Dans les ovaires, on trouve une prolifération des cellules de la granulosa et de la thèque [1]. Ces résultats suggèrent que l'inhibine et l'activine sont des facteurs respectivement freinateurs et stimulateurs de la croissance de certaines cellules gonadiques, par un mécanisme autocrine dans le cas des cellules de Sertoli et de la granulosa, et par un mécanisme paracrine dans les cellules thécales. D'une certaine manière, on pourrait considérer le gène de l'inhibine comme un anti-oncogène pour ses cellules cibles. L'intervention d'anomalies de ce système dans des tumeurs gonadiques spontanées reste à prouver, de même que l'éventuelle utilité d'inhibine recombinante dans certains cancers des testicules et de l'ovaire dans l'espèce humaine.

[1. Matzuk MM, et al. Nature 1992 ; 360 : 313-19.]

Le gène codant pour la protéinase- 3 est localisé sur le chromosome 19. La protéinase-3 (PR-3) est une sérine protéinase neutre d'environ 26000 daltons localisée dans les granulations azurophiles des polynucléaires neutrophiles humains. Elle est identique à la myéloblastine, identifiée pour la première fois dans une lignée de cellules leucémiques, et elle représente l'auto-antigène principal associé à la granulomatose de Wegener, à l'origine de la formation des anticorps correspondants (voir $\mathrm{m} / \mathrm{s}$ $n^{\circ}$ 8, vol. 8, p. 927). A.B. Sturrock et al. [1] ont cloné et séquencé le gène codant pour la PR-3. Le gène comporte environ 6500 paires de bases 
et est constitué de cinq exons et de quatre introns. Son organisation est identique à celle des gènes des autres sérines protéases. Cependant il est plus grand que les gènes de celles-ci, du fait de la taille accrue des introns I et III. Les gènes de la PR-3, de l'élastase leucocytaire et de la cathepsine $G$ ont probablement un ancêtre commun. Le gène de la PR-3 a été localisé sur le chromosome 19. Les auteurs rappellent que la PR-3 pourrait être impliquée dans la prolifération et la différenciation des cellules myéloïdes. Il est intéressant de noter que des anomalies portant sur le chromosome 19 ont été rapportées dans les leucémies aiguës myéloïdes et chez $18 \%$ des malades ayant une leucémie myéloïde chronique en transformation aiguë myéloblastique. [1. Sturrock AB, et al. J Biol Chem 1992 ; 267 : 21193-9.]

प Les armes secrètes des myxomavirus : de faux récepteurs de cytokines. Le myxomavirus de type sauvage est un poxvirus extrêmement virulent chez les lapins adultes domestiques. Parmi ses gènes de virulence, la séquence ouverte $M-T 7$ code pour une protéine soluble qui a une ressemblance significative avec le récepteur de l'interféron $\gamma$. L'équipe canadienne d'Edmonton à l'origine de cette découverte [1] montre que la protéine virale M-T7 est effectivement capable de fixer l'interféron $\gamma$. Par conséquent, la sécrétion par les cellules infectées d'un analogue soluble non fonctionnel du récepteur de l'interféron $\gamma$ empêche cette cytokine de manifester son puissant effet antiviral en se fixant à ses récepteurs cellulaires normaux. Cette stratégie est également utilisée par un autre poxvirus, le virus du fibrosarcome de Shope, dont l'un des gènes de virulence ressemble à un récepteur du facteur TNF (tumor necrosis factor). Ce dernier gène semble protéger des cellules infectées contre l'action des interférons $\alpha$ et $\beta$. Les auteurs canadiens proposent d'appeler " virorécepteurs " ces gènes de virulence agissant en divertissant des cytokines antivira$\mathrm{m} / \mathrm{s} n^{\circ} 1$ vol. 9, janvier 93 les de leur fonction physiologique normale.

[1. Watkins DC, et al. Science 1992 258 : 1373-5.]

$\square$ Les lymphocytes $T$ des cancéreux ne répondent plus! De nombreux travaux ont tenté de comprendre les mécanismes des déficits immunitaires acquis observés chez les porteurs de cancers, souris ou humains. Dans certains cas une insuffisance de présentation des molécules du complexe majeur d'histocompatibilité a pu être mise en cause, notamment dans les tumeurs expérimentales provoquées par les souches oncogéniques d'adénovirus. D'autres fois, le désordre semble intrinsèque aux lymphocytes $T$. L'équipe de A.C. Ochoa (NCI, Frederick, MD, USA), vient de montrer que les lymphocytes de souris cancéreuses ne répondaient pas à une stimulation par un anticorps anti-CD3. CD3 est un complexe multimoléculaire, associé au récepteur de l'antigène des lymphocytes $\mathrm{T}$, composé normalement de quatre types de chaînes, $\gamma, \delta, \epsilon$ et $\zeta$. Le complexe $\mathrm{CD} 3$ joue un rôle très important dans la transduction du signal d'activation, agissant notamment par l'intermédiaire de protéines kinases cytoplasmiques spécifiques des résidus tyrosine, produits des protooncogènes $p 56^{\text {Ick }}[1]$ et $p 59^{f y n}$ [1]. $H$. Mizoguchi et al. ont observé que ces lymphocytes $\mathrm{T}$ non réactifs des souris tumorales ne possédaient pas de protéines p56 $6^{\text {lck }}$ et p59.ton détectables, alors que les messagers codant pour ces protéines étaient normalement synthétisés. Cette absence des tyrosine kinases associée à la transduction du signal d'activation des lymphocytes $\mathrm{T}$ pourrait être secondaire à un déficit en sous-unités $\zeta$, elles aussi à peine détectables dans ces lymphocytes alors que leur messager est normal. Il est possible que des sousunités $\gamma$, identiques à celles du récepteur pour le fragment Fc des immunoglobulines E [2], remplacent ici les sous-unités $\zeta$ pour stabiliser le récepteur $\mathrm{T}(\alpha, \beta)$, mais soient incapables d'être normalement couplées aux pro- téines kinases $\mathrm{p} 56^{\text {Ick }}$ et $\mathrm{p} 59^{\text {fyn }}$ qui seraient alors dégradées [3]. Les auteurs supposent que les cellules cancéreuses agissent sur la stabilité des sous-unités $\zeta$ par un facteur soluble qui reste, à ce jour, parfaitement spéculatif.

[1. Fagard R. médecine/sciences 1989 ; 5 : 554-60.]

[2. Rochet $\mathbf{N}$, et al. médecine/sciences $1992 ; 8$ : 359-67.]

[3. Mizoguchi H, et al. Science 1992 ; 258 : 1795-8.]

Effet in vivo d'un oligonucléotide anti-sens anti NF- $\kappa$ B sur une tumeur transplantée de souris transgéniques HTLV1-Tax. Les souris transgéniques exprimant le gène tax du virus HTLV1 sous le contrôle du LTR de ce virus développent des fibrosarcomes transplantables. En culture, les cellules tumorales synthétisent la protéine Tax et, également, de grandes quantités du facteur de transcription NF- $\mathrm{KB}$. I. Kidajima et al. (La Jolla, CA, USA) ont démontré que des oligonucléotides anti-sens anti-Tax n'ont pas d'effet sur ces cellules alors que des oligo-nucléotides anti $\mathrm{NF}-\kappa \mathrm{B}$ en bloquent la croissance. In vivo, l'administration intrapéritonéale de $40 \mu \mathrm{g}$ d'oligonucléotides anti-sens anti-NF- $\kappa$ B par gramme de poids de souris, dose répétée trois jours après la première injection, inhibe la croissance d'une tumeur transplantée sept jours auparavant à la base du membre postérieur. Là aussi, les oligonucléotides anti-Tax sont inactifs. Ce remarquable résultat souligne l'intérêt d'une thérapeutique dirigée spécifiquement contre NF- $\kappa \mathrm{B}$ dans les tumeurs provoquées par HTLV-1, par exemple les leucémies T. L'effet transformant de ces virus pourrait en effet passer par la production continue de $\mathrm{NF}-\kappa \mathrm{B}$. De plus, ces résultats s'ajoutent au petit nombre de cas où un effet in vivo d'oligonucléotides antisens a pu être démontré.

[1. Briand P. médecine/sciences 1992 ; 8 : 208-13.]

[2. Kidajima I, et al. Science 1992 ; 258 : 1792-5.] 